\title{
The Oblique Pulsator Model for the Blazhko Effect in RR Lyrae Stars. Theory of Amplitude Modulation I.
}

\author{
Hiromoto Shibahashi \\ Department of Astronomy, University of Tokyo, Tokyo 113-0033, Japan
}

\begin{abstract}
By assuming that RR Lyrae stars have fairly strong dipole magnetic fields with symmetry axis oblique to the rotation axis of the star, I show that the oscillation mode which would be a pure radial oscillation in absence of the magnetic field has a quadrupole component, whose symmetry axis coincides with the magnetic axis. The aspect angle of the quadrupole component changes due to the stellar rotation, and this apparent amplitude variation is interpreted as the Blazhko effect in RR Lyrae stars. It is shown that, in the case of off-axis magnetic field, phase modulation is expected. I list up some theoretical predictions based on this model, which would observationally examine this hypothesis.
\end{abstract}

\section{Introduction}

In about $20 \%$ of RR Lyrae stars, amplitudes of pulsations in the light curve are known to be modulated with periods much longer than the pulsation periods themselves. This phenomenon is eponymously called the Blazhko effect. Recent analyses of the Blazhko stars from the MACHO database have revealed that some of the Blazhko stars show nearly pure amplitude modulation while some others show phase modulation (Kurtz et al. 2000). In this paper, I assume that RR Lyrae stars have substantial magnetic fields and that the magnetic symmetry axis is inclined to the rotation axis of the star, and I propose the oblique pulsator model for the Blazhko effect. This is based on the thought that the modulation periods are of the same order of the plausible rotation periods of these stars (Christy 1966). This model for the amplitude modulation is essentially the same as the oblique pulsator model for the rapid oscillations of the Ap stars (Kurtz 1982), and the obliquely oscillating magnetic rotator model proposed by Cousens (1983). I will show that, in the case of an off-axis magnetic field, oscillation components having a phase difference of $90^{\circ}$ appear and this phase difference induces the phase modulation.

\section{Amplitude Modulation}

I assume that the star is rigidly rotating (due to the presence of the strong magnetic field) with angular frequency $\Omega$, and that the star has a dipole magnetic field whose symmetry axis passes through the stellar center (on-axis), and I consider the oscillations in the co-rotating frame. I set the temporal dependence of the eigenfunctions as $\exp (i \omega t)$ in the rotating frame. I hereafter treat the 

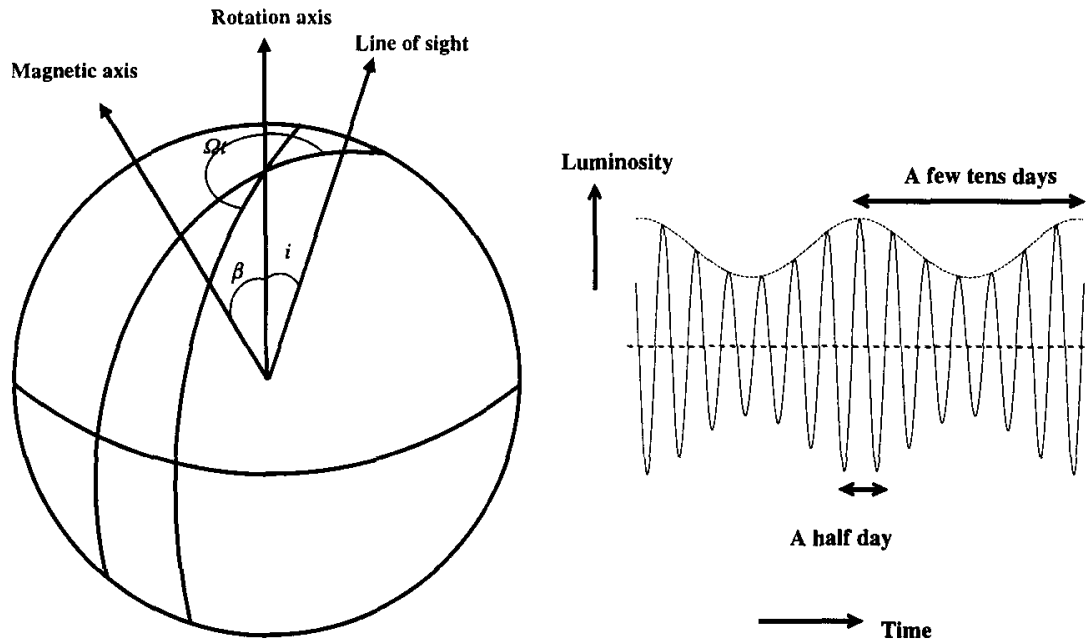

A half day

Figure 1. (Left) Geometry of the oblique rotator and (Right) a schematic expected light curve.

Lorentz force and Coriolis force as perturbations, and assume the Lorentz force is stronger than the Coriolis force. Being influenced by such a strong magnetic field, the eigenmode, which would be a pure radial mode in the absence of a magnetic field, is deformed to have an axially symmetric quadrupole component, whose symmetry axis coincides with the magnetic axis. Hence, the eigenfunction at the surface is characterized by means of a superposition of the spherical harmonic with $\ell=m=0$ and that of $\ell=2$ and $m=0$ with respect to the magnetic axis (Shibahashi 1995, Shibahashi \& Takata 1995); i.e.,

$$
\left[Y_{0}^{0}\left(\theta_{B}, \phi_{B}\right)+\alpha Y_{2}^{0}\left(\theta_{B}, \phi_{B}\right)\right] \exp (i \omega t),
$$

where $\alpha$ is determined by the magnetic field of the star and the unperturbed eigenfunction of the mode. Since I am assuming that the magnetic axis is oblique to the rotation axis, the aspect angle of the pulsation axis varies with the rotation of the star. Therefore, the contribution of the quadrupole component of the eigenfunction to the apparent intensity variation changes with time.

The observable variation is obtained by integrating equation (1) over the visible disc. Note that a spherical harmonic expressed by the spherical coordinates with respect to the magnetic axis $\left(\theta_{B}, \phi_{B}\right)$ is written in terms of $(2 \ell+1)$ spherical harmonics of the same degree $\ell$ with respect to the spherical coordinates $\left(\theta_{L}, \phi_{L}\right)$ :

$$
Y_{\ell}^{m}\left(\theta_{B}, \phi_{B}\right)=\sum_{m^{\prime}=-\ell}^{\ell} \sum_{m^{\prime \prime}=-\ell}^{\ell}(-1)^{m^{\prime}} d_{m m^{\prime}}^{(\ell)}(\beta) d_{m^{\prime} m^{\prime \prime}}^{(\ell)}(i) Y_{\ell}^{m^{\prime \prime}}\left(\theta_{L}, \phi_{L}\right) \exp \left(-i m^{\prime} \Omega t\right),
$$

where $\left\{d_{m m^{\prime}}^{(\ell)}(\beta)\right\}$ and $\left\{d_{m^{\prime} m^{\prime \prime}}^{(\ell)}(i)\right\}$ are the matrices to transform the spherical harmonics expressed in terms of the spherical coordinates with respect to the 
magnetic axis, to those expressed in terms of the spherical coordinates with respect to the line-of-sight. The angles $\beta$ and $i$ are the angle between the magnetic axis and the rotation axis and that between the line-of-sight and the rotation axis, respectively (see Fig. 1). Integrating equation (1) over the visible disc $\left(\theta_{L}=[0, \pi / 2]\right.$ and $\left.\phi_{L}=[0,2 \pi]\right)$ after substituting equation (2) into equation (1) leads to a quintuplet fine structure with a spacing equal to the rotation frequency in the power spectrum (see Fig. 2). Since the integral with respect to $\phi_{L}$ becomes zero unless $m^{\prime \prime}=0$, the amplitude of the component at $\omega+m^{\prime} \Omega$ is proportional to $d_{0 m^{\prime}}^{(2)}(\beta) d_{m^{\prime} 0}^{(2)}(i)$ (Shibahashi 1986). The relative ratios of the amplitudes of the side-peaks to the amplitude of the central peak depend on the strength of the magnetic field. On the other hand, the relative ratios among the side-peak amplitudes are dependent on the geometrical configuration of the star - that is, the angles $\beta$ and $i$ (see Fig. 1). By analyzing the amplitude ratios, we can determine these angles. If we write the amplitude of the component at $\omega+m^{\prime} \Omega$ as $A_{m^{\prime}}$, then $\left(A_{2}+A_{-2}\right) /\left(A_{1}+A_{-1}\right)$ is given by (Shibahashi 1986)

$$
\frac{A_{2}+A_{-2}}{A_{1}+A_{-1}}=\left|\frac{d_{02}^{(2)}(\beta) d_{20}^{(2)}(i)}{d_{01}^{(2)}(\beta) d_{10}^{(2)}(i)}\right|=\frac{1}{4}|\tan \beta \tan i| .
$$

Since the function $\tan \beta \tan i$ tends to be significantly larger than four for a wide range of $\beta$ and $i$, equation (3) implies that, in many cases, the fine structure looks like a triplet rather than a quintuplet. Fourier analyses of some of the Blazhko stars show a triplet fine structure, and it is consistent with the above prediction (Borkowski 1980; Smith et al. 1994; Kovács 1995; Kolenberg et al. 2000). The apparent magnetic field strength should also vary with the rotation of the star, and $\left(1-B_{\min } / B_{\max }\right) /\left(1+B_{\min } / B_{\max }\right)$ should have the same dependence on $\beta$ and $i$ as the amplitude ratio $\left(A_{2}+A_{-2}\right) /\left(A_{1}+A_{-1}\right)$, where $B_{\min }$ and $B_{\max }$ denote the apparent minimum field strength and the maximum field strength, respectively; that is,

$$
\frac{A_{2}+A_{-2}}{A_{1}+A_{-1}}=\left(1-\frac{B_{\min }}{B_{\max }}\right) /\left(1+\frac{B_{\min }}{B_{\max }}\right) .
$$

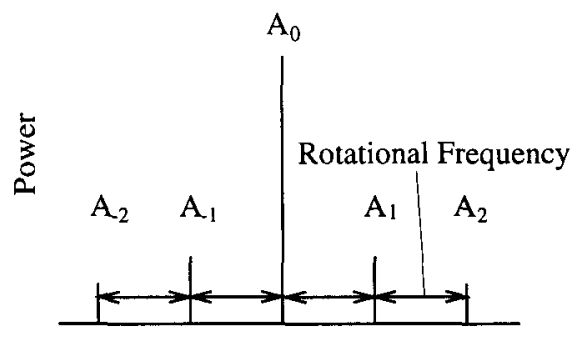

Frequency

Figure 2. Schematic power spectrum expected from the oblique pulsator model. 


\section{Phase Modulation}

In the case of an off-axis dipole magnetic field, the eigenfunction is deformed to have $\ell=2$ and $m \neq 0$ components with respect to the axis which is parallel to the magnetic axis and passes the stellar center:

$$
\left[Y_{0}^{0}\left(\theta_{B}^{\prime}, \phi_{B}^{\prime}\right)+\alpha Y_{2}^{0}\left(\theta_{B}^{\prime}, \phi_{B}^{\prime}\right)+\alpha^{\prime} Y_{2}^{1}\left(\theta_{B}^{\prime}, \phi_{B}^{\prime}\right)+\alpha^{\prime \prime} Y_{2}^{-1}\left(\theta_{B}^{\prime}, \phi_{B}^{\prime}\right)\right] \exp (i \omega t) .
$$

Rewriting $Y_{2}^{m}\left(\theta_{B}^{\prime}, \phi_{B}^{\prime}\right)$ in terms of $Y_{2}^{m^{\prime \prime}}\left(\theta_{L}, \phi_{L}\right)$ and integrating over the visible disc leads not only to the components proportional to $\cos m^{\prime} \Omega t$, but also to those proportional to $\sqrt{-1} \sin m^{\prime} \Omega t$. This induces the variable components whose phases are differ from each other by $90^{\circ}$, and thus eventually to the phase modulation;

$$
\Delta L \propto a(\Omega t) \cos (\omega t)+b(\Omega t) \sin (\omega t) \propto A(\Omega t) \cos [\omega t+\phi(\Omega t)] .
$$

\section{RR Lyrae Stars As a Nonradial Pulsator}

The remaining problem is to estimate what strength magnetic fields are required to explain the observed Blazhko amplitude. To see this, one has to determine the expansion coefficient $\alpha$ in equation (1), which is dependent on the magnetic field strength. It should be remembered that nonradial p-mode oscillations in giant stars, such as RR Lyrae stars, have a dual character; that is, they behave like gravity waves in the deep interior, while they have properties of acoustic waves in the external regions (cf. Unno et al. 1989). Hence, the nonradial oscillation $\mathrm{p}$ modes of $\ell=2$ have a gravity wave character in the deep interior. Because of the high central condensation as a result of stellar evolution, the number of nodes in the g-zone of RR Lyrae stars is quite large, and the wavelength is very short there. Because the gravity waves with such a short wavelength must be damped by nonadiabatic effects, the wave that propagates from the outer p-mode regions to the inner $\mathrm{g}$-mode regions will never return. I have solved the wave equation by taking this effect into account by imposing the boundary condition of the progressive wave, whose energy is transmitted downward at the bottom of the p-mode regions, and determined the expansion coefficient $\alpha$. In the framework of the linear theory, despite of energy leakage due the gravity wave, the star is still pulsationally unstable, because the $\ell=2$ component of the eigenfunction deformed by the magnetic field is relatively much smaller than the radial component (i.e., $|\alpha| \ll 1$ ), and the excitation of the radial component due to the $\kappa$ mechanism dominates over the energy leakage.

\section{Numerical Simulation for Amplitude Modulation}

Since the existence of magnetic fields in RR Lyrae stars is still controversial, the magnetic field strength is treated as a free parameter in the following calculation. As the first step, I assume an on-axis magnetic field. The numerical results shown in Fig. 3 are obtained under the condition of the magnetic field strength set to $1000 \mathrm{G}$, and one can see the dependence of luminosity modulation on the geometrical configuration (Shibahashi 1995). From the top to the bottom, the 


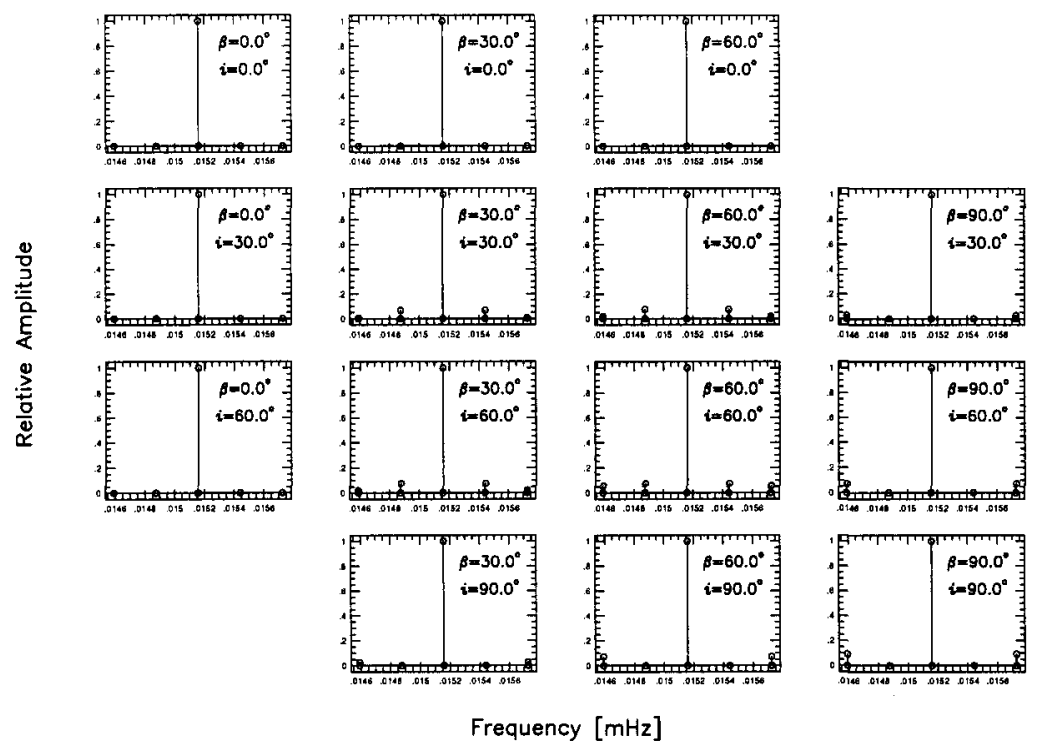

Figure 3. Geometrical dependence of the power spectrum.

angle $i$ is $0^{\circ}, 30^{\circ}, 60^{\circ}$, and $90^{\circ}$, and from the left to the right, the angle $\beta$ is $0^{\circ}, 30^{\circ}, 60^{\circ}$, and $90^{\circ}$. The luminosity variation becomes a double wave when $\beta+i>90^{\circ}$.

Next, I have fixed the geometrical configuration of the star and have changed the magnetic field strength to see the dependence of the luminosity variation on the magnetic strength. From this investigation, it has been shown that a magnetic field of order $1 \mathrm{kG}$ is sufficient to explain the observed Blazhko effect of RR Lyrae stars (Shibahashi 1995).

\section{Discussion}

The present oblique pulsator model yields the following predictions, by which observational checks can be done:

- In the case of Blazhko stars with amplitude modulation, an equally-spaced quintuplet fine structure is expected.

- The side components of the quintuplet (or triplet) are due to the $\ell=2$ component of the eigenfunction.

- There must exist a substantial magnetic field, varying with the Blazhko period.

- In the case of Blazhko stars with amplitude modulation, the geometrical configuration, $\tan \beta \tan i$, should be consistent with the magnetic field variation.

- In the case of Blazhko stars showing phase modulation, the magnetic field is an off-axis field. 
- The rotation period is identical to the Blazhko period.

- Since the amplitude variation is not a result of the appearance of new modes, but a consequence of deformation of the eigenfunction, it is expected that the highest amplitude of the Blazhko RR Lyrae stars should fit with the period-amplitude relation of the normal RR Lyrae stars.

So far, analyses of some of the Blazhko RR Lyrae stars have shown equallyspaced triplet fine structure, but a quintuplet fine structure has not yet been detected. To detect the undetected side components, high S/N observations are necessary. As for the magnetic field, there have been observational reports of detection of the magnetic field of RR Lyrae itself (e.g., Romanov et al. 1987), but they are not well-confirmed. Observational tests for the predicted magnetic field of RR Lyrae stars are highly desired, since the presence of the magnetic field is the key assumption of the present model. As for the period-amplitude relation, it is known that the highest amplitude of the Blazhko RR Lyrae stars fits with the period-amplitude relation of the singly periodic $R R$ Lyrae stars (Szeidl 1988).

Acknowledgments. This work was supported in part by a Grant-in-Aid for Scientific Research of the Japan Society for the Promotion of Science (No. 11440061).

\section{References}

Borkowski, K. J. 1980, Acta Astron., 30, 393

Christy, R. F. 1966, ApJ, 144, 108

Cousens, A. 1983, MNRAS, 203, 1171

Kolenberg, K., Aerts, C., Chadid, M., \& Gillet, D. 2000, in these proceedings, p. 286

Kovács, G. 1995, A\&A, 295, 693

Kurtz, D. W. 1982, MNRAS, 200, 807

Kurtz, D. W. and the MACHO collaboration 2000, in these proceedings, p. 291

Romanov, Yu. S., Udovichenko, S. N., \& Frolov, M. S. 1987, Soviet Ast. Letters, 13,29

Shibahashi, H. 1986, in Hydrodynamic and Magnetohydrodynamic Problems in the Sun and Stars, ed. Y. Osaki (Tokyo: Univ. of Tokyo), 195

Shibahashi, H. 1995, in ASP Conf. Ser. Vol. 76, GONG '94: Helio- and Asteroseismology from the Earth and Space, ed. R. K. Ulrich, E. J. Rhodes, Jr., \& W. Däppen (San Francisco: ASP), 618

Shibahashi, H., \& Takata, M. 1995, in ASP Conf. Ser. Vol. 83, Astrophysical Applications of Stellar Pulsation, ed. R. Stobie \& P. Whitelock (San Francisco: ASP), 42

Smith, H., Matthews, J. M., Lee, K. M., Williams, J., Silberman, N. A., \& Bolte, M. 1994, AJ, 107, 679

Szeidl, B. 1988, in Multi-mode Stellar Pulsations, ed. G. Kovács, L. Szabados, \& B. Szeidl (Budapest: Konkoly Observatory), 45

Unno, W., Osaki, Y., Ando, H., Saio, H., \& Shibahashi, H. 1989, Nonradial Oscillations of Stars (Tokyo: Univ. of Tokyo Press) 


\section{Discussion}

Géza Kovács: What happens to the magnetic field of an oblique rotator (i.e. Blazhko star in your model) if you move the model toward higher temperature, and as a result of it the star becomes a first overtone pulsator? I think that the low percentage of the Blazhko RRc stars recently discovered in the LMC and in other places (see these proceedings) implies that the magnetic field should become in general very weak. Do you think this is possible?

Hiromoto Shibahashi: At this moment, I do not have any reason to believe that the magnetic field of the Blazhko stars becomes weaker (or stronger) with the evolutionary change in temperature. Since RRc stars have smaller amplitudes than RRab stars, qualitatively speaking, I think that the detectability of the amplitude modulation in RRc type stars would be lower than in the case of RRab stars.

Douglas Gough: My comment is that if the Coriolis force (in the rotating frame) is not negligible compared with the Lorentz force, then if the axes of rotation and the magnetic field were to differ, as you have supposed, there would be an asymmetry in amplitude (and frequency) of the multiplet structure of the power spectra.

My question is whether the necessary periodic variation in the magnetic field is observed to have a period identical with that of the Blazhko variation.

Hiromoto Shibahashi: Your comment is right. Anyway, in my model, the Blazhko period (about a few tens of days) is identical with the rotation period of the star, and then the Coriolis force is negligibly small compared with the Lorentz force as far as we assume that the magnetic field is of the order of $1 \mathrm{kG}$.

Such periodic variation in the magnetic field was reported by Romanov et al. 1987, Sov. Astron. Lett., 13, 29) in the case of RR Lyrae itself. But there is no other observation to confirm that.

Merieme Chadid: 1. For your model, do you need a variable magnetic field during the pulsation cycle or the Blazhko cycle?

2. For the explanation of the Blazhko effect, what do you think about the resonance models by Van Hoolst and Cox?

Hiromoto Shibahashi: 1. My model predicts that the magnetic field strength apparently varies with the Blazhko phase but not with the phase of the pulsation. 2. I would like to postpone my privilege to answer your second question till we hear Van Hoolst's talk. I would like to know how he explains the amplitude and phase modulation, and how his model can be justified observationally.

Luis Balona: The nonlinear effects almost completely dominate the light curves of these stars and determine the amplitudes of the equidistant frequency components. It would be necessary to produce a model for the nonlinearities before one can test the effect that you are suggesting.

Hiromoto Shibahashi: It is obvious that the non-sinusoidal shape of the light curves of pulsation is a result of the nonlinear effects. I think that the same is true for the asymmetry of the maximum amplitude modulation and the min- 
imum amplitude modulation in the Blazhko effect. There is no doubt that we have to take into account the nonlinear effects to explain these features. However, many fundamental features of stellar pulsation, such as the pulsation periods, are well described even by the linear theory. I think that, at the very initial stage of finding a solution, it is necessary to simplify the problem and to find an easy solution first. So, I think that, at this stage, we may start from the linear theory to find a plausible explanation for some fundamental features of the Blazhko effect, such as the Blazhko period and the equidistant multiplet in the power spectrum.

Darragh O'Donoghue: Is your model able to explain phase variations?

Hiromoto Shibahashi: I have just heard about phase variations from Don Kurtz's talk, and so I have not prepared for any explanation about phase variations. I will think about how to explain phase variations in the framework of my model. 